\title{
Pseudomonas aeruginosa: distribution and antibiotic profile of one of the ESKAPE pathogen
}

\author{
Karthick M. ${ }^{1}$ \\ ${ }^{1}$ Dr. Mowna Karthick, Lab Specialist, Dr. Sulaiman Al Habib Hospital, Riyadh, Saudi Arabia.
}

Corresponding Author: Dr. Mowna Karthick, Lab Specialist, Dr. Sulaiman Al Habib Hospital, Riyadh, Saudi Arabia. Email: drmowna@gmail.com

\begin{abstract}
Background: Pseudomonas aeruginosa is an opportunistic gram-negative pathogen known for its ingenious mode of infection. The management of infections with Pseudomonas has been quite a challenge. The bacteria have intrinsic resistance against most of the routine antibiotics. Such a scenario places the health care delivery system at a challenging end point with very minimal options of care and increased rates of morbidity and mortality. This study was done to assess the pattern of presentation of Pseudomonas aeruginosa in hospital settings. Methods: This cross-sectional study was carried out among 280 specimens which were isolated for a period of 17 months in our tertiary care hospital. The blood culture bottles were placed in Bac T/ Alert $3 \mathrm{D}$ and the positive culture bottle was processed by Grams stain and in routine bacteriological media for inoculation and incubated. Pseudomonas aeruginosa organisms isolated from all the clinical samples were subjected for determining the identification and antibiotic susceptibility profile by VITEK 2 and manual methods. Results: A total of 280 samples were analyzed in this study. The background characteristics of the specimens analyzed is given in table 1. In this study, majority of the samples were from inpatients (71.4\%). Among the total samples, most specimens were urine samples $(37.5 \%)$, followed by pus $(23.1 \%)$. The organisms showed high sensitivity to Amikacin, Ceftazidime and Gentamycin (98\%). Conclusion: From the present findings, we understand that Amikacin and Gentamycin can be the proposed drug of choice for severe infections with Pseudomonas aeruginosa.
\end{abstract}

Keywords: Antimicrobial resistance, Carbepenems, Fluoroquinolones, Pseudomonas aeruginosa

\section{Introduction}

Pseudomonas aeruginosa is an opportunistic gramnegative pathogen known for its ingenious mode of infection. The mechanism of action of the bacteria is by production of exotoxins inside the host body which blocks protein synthesis by binding to the coenzyme Nicotinamide Adenine Dinucleotide (NAD) followed by the release of nicotinamide, which significantly damages oxidation reduction reactions and protein synthesis. It also invades the immune system by inactivating the complement cascade [1]. Pseudomonas is capable of surviving in a variety of environmental conditions as it requires minimum oxygen and nutrition.

It has been strongly associated with severe lifethreatening infections in immunocompromised individuals, apart from wound infections in burns patients and other secondary infections in patients with diabetes mellitus, etc [2]. The management of infections with Pseudomonas has been quite a challenge.

Manuscript received: $20^{\text {th }}$ August 2019

Reviewed: $30^{\text {th }}$ August 2019

Author Corrected: $7^{\text {th }}$ September 2019

Accepted for Publication: $11^{\text {th }}$ September 2019
The bacteria have intrinsic resistance against most of the routine antibiotics. For many years, Carbapenem was considered as the drug of choice, however inappropriate use and misuse of the drug has resulted in drug resistance in clinical settings [3]. The mechanism of resistance is either plasmid mediated or chromosome mediated. Plasmid mediated resistance act by hydrolyzing imipenem and meropenem [4]. Chromosome mediated resistance is achieved by the loss of porin and overexpression of efflux pumps [5]. In such scenario Carbepenem resistant bacteria are generally resistant to most antimicrobials except polymyxins and tigecyclines [6]. Considering the high rate of toxicity and suboptimal pharmacokinetics, the use of polymyxins and tigecyclines are restricted [7].

The existing resistance mechanisms has resulted in the formulation of multidrug resistance P.aeruginosa phenotype which is defined as a bacterium which is resistant to antimicrobial agents which are included in three or more antipseudomonal anti-microbial classes namely carbepenem, fluoroquinolones, penicillins/ 
cephalosporines aminoglycosides [8]. Such a scenario places the health care delivery system at a challenging end point with very minimal options of care and increased rates of morbidity and mortality.

\section{Objectives}

This study was carried out to-

1. Observe the pattern of distribution of $P$. aeruginosa among the clinical specimens

2. Analyze the antibiotic sensitivity pattern in the isolates.

\section{Methodology}

Study setting: This study was carried out in the Microbiology laboratory of our tertiary care hospital in Riyadh, Saudi Arabia.

Duration of study: The study was carried out for a period of 17 months from January 2017 to May 2018.

Type of study: Cross sectional study

Sampling methods: The study samples were selected by convenient sampling

Sample size calculation: Based on intensive literature review, the lowest prevalence of Pseudomonas infections was observed to be $7-10 \%$ in urinary tract infections. [9] Therefore, assuming $7 \%$ as the lowest prevalence, at $95 \%$ confidence limits and 3\% absolute precision, the sample size was calculated as 277.76 and was rounded off to 280 .

\section{Original Research Article}

Inclusion criteria: All the microbiological samples received during this period from both outpatients and inpatients were examined. Cultures positive for Pseudomonas aeruginosa were included in the study.

Exclusion criteria: All Samples which were repeated from the same patient and same site of collection were excluded.

Data collection procedure: The blood culture bottles were placed in Bac T/ Alert $3 \mathrm{D}$ and the positive culture bottle was processed by Grams stain and in routine bacteriological media for inoculation and incubated. All other samples were inoculated in the respective media and methods as per standard guidelines and incubated.

Pseudomonas aeruginosa organisms isolated from all the clinical samples were subjected for determining the identification and antibiotic susceptibility profile by VITEK 2 and manual methods.

Data analysis: Data was entered and analyzed using Microsoft Excel spreadsheet 2010. The distribution and pattern of sensitivity of $P$. aeruginos $a$ was expressed as percentages.

Ethical consideration and permission: Approval was obtained from the Institutional Ethics Committee prior to the commencement of the study. Each participant was explained in detail about the study and informed consent was obtained prior to the sample collection.

\section{Results}

A total of 280 samples were analyzed in this study. The background characteristics of the specimens analyzed is given in table 1. In this study, majority of the samples were from inpatients (71.4\%). Among the total samples, most specimens were urine samples $(37.5 \%)$, followed by pus $(23.1 \%)$.

Table-1: Background characteristics of the specimens analyzed:

\begin{tabular}{|c|c|c|c|}
\hline S. No. & Characteristics & Frequency $(\mathrm{N}=\mathbf{2 8 0})$ & Percentage (\%) \\
\hline \multirow[t]{3}{*}{1} & \multicolumn{3}{|l|}{ Source of specimens } \\
\hline & Outpatients & 80 & 28.6 \\
\hline & Inpatients & 200 & 71.4 \\
\hline \multirow[t]{9}{*}{2} & \multicolumn{3}{|l|}{ Type of specimen } \\
\hline & Pus & 65 & 23.1 \\
\hline & Urine & 105 & 37.5 \\
\hline & Blood & 15 & 5.4 \\
\hline & Tracheal aspiration & 17 & 6.1 \\
\hline & Ear discharge & 11 & 3.9 \\
\hline & Sputum & 61 & 21.8 \\
\hline & Pleural fluid & 3 & 1.1 \\
\hline & Conjunctival smear & 3 & 1.1 \\
\hline
\end{tabular}


Original Research Article

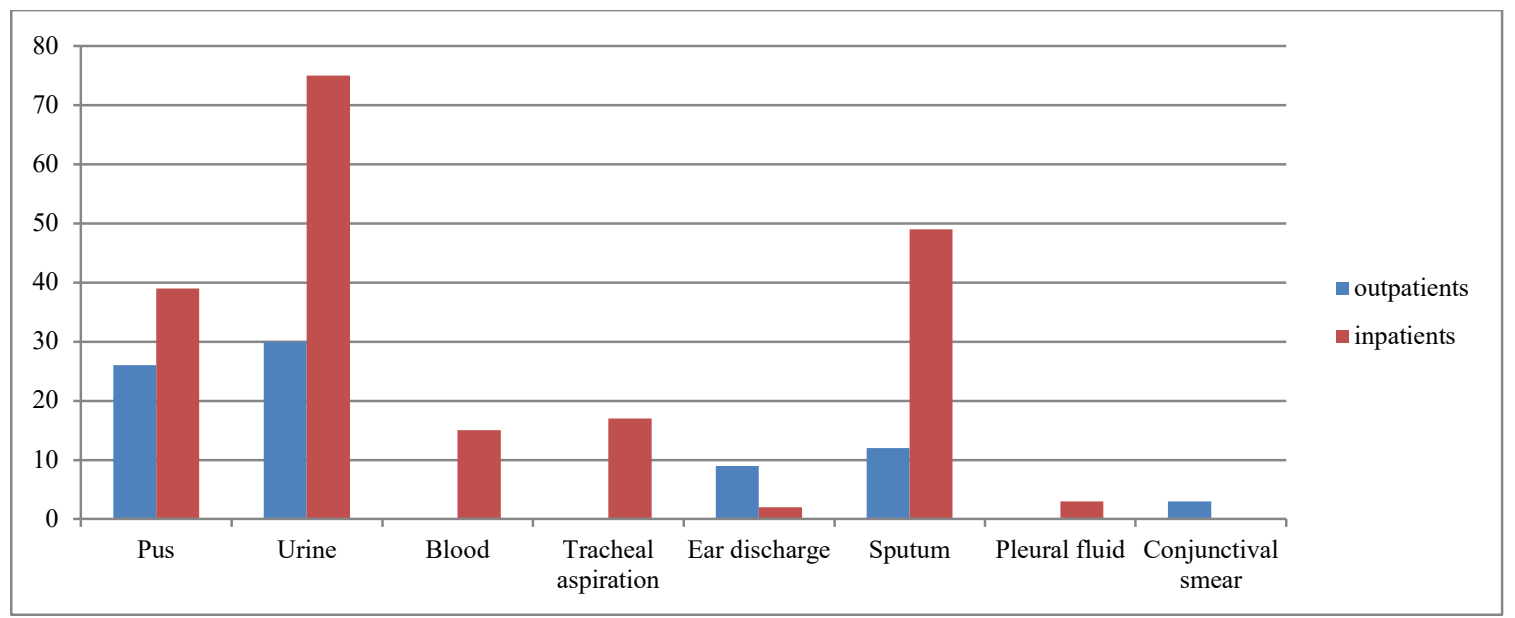

Figure-1: Comparison of isolates between outpatients and inpatients

Table-2: Antibiotic sensitivity pattern among the isolates:

\begin{tabular}{|c|c|c|c|}
\hline S. No & Antibiotic & Outpatients (\%) & Inpatients (\%) \\
\hline 1 & Amikacin & 98 & 95 \\
\hline 2 & Gentamycin & 97 & 92 \\
\hline 3 & Ceftazidime & 98 & 85 \\
\hline 4 & Cefepime & 98 & 87 \\
\hline 5 & Ciprofloxacin & 83 & 77 \\
\hline 6 & Levofloxacin & 83 & 77 \\
\hline 7 & Piperacillin+ Tazobactam & 94 & 87 \\
\hline 8 & Imipenem & 93 & 84 \\
\hline 9 & Meropenem & 94 & 84 \\
\hline 10 & Aztreonam & 88 & 78 \\
\hline
\end{tabular}

The comparison of the isolates between outpatients and inpatients is given in figure 1. Among the inpatients, the majority of the samples were urine, followed by pus and sputum. Among the inpatients, the highest samples were from urine and pus.

The results of antibiotic sensitivity testing are given in table 2. As far as the outpatient samples were analyzed $98 \%$ sensitivity was found with Amikacin, Ceftazidime and Cefipime. With inpatient samples, sensitivity was found to be highest (95\%) with Amikacin, followed by Gentamycin (92\%).

\section{Discussion}

Pseudomonas aeruginosa is one of the most common gram-negative infections resulting in multidrug resistance and hospital acquired infections. Apart from urinary tract infections, several practices including prolonged hospital stay, ICU admissions, tracheal intubations, etc are considered as proven risk factors for P.aeruginosa infections [10]. Our study was done to evaluate the characteristic profile of P.aeruginosa infections in hospital settings. We observed that majority of the isolates were from inpatients, especially with urine samples. Djordjevic Z et al in his study reported that 7$10 \%$ of all urinary tract infections are caused by P.aeruginosa [9]. Psuedomonas causing urinary tract infections are often a resultant of cathether associated urinary tract infections (CAUTI) which accounts for $40 \%$ of all nosocomial infections [11]. These infections are associated with a high 30-day mortality of $17.7 \%$. Moreover, the sensitivity of these bacteria towards antibiotics were as low as $69 \%$ for piperacillin and tazobactam [12].

Our study also demonstrated a higher antibiotic sensitivity to Amikacin for both outpatients and inpatients and Gentamycin for inpatient infections. A 
Original Research Article

study done by Golia $\mathrm{S}$ et al also reported similar findings [13]. Another study done by Javiya VA et al demonstrated high rates of sensitivity to Cefipime, which correlated with our findings in outpatient samples [14]. The antibiotic resistance observed in Pseudomonas could be due to synergy between type $1 \mathrm{AmpC}$ beta-lactamase and low outer membrane permeability [15].

In our study, high level of resistance was observed with fluoroquinolones namely ciprofloxacins and levofloxacin. Moreover, the presence of MDR rate (resistance to three antipseudomonals) was quite low (18\%). A study done by Golia et al also reported similar finding of $10 \%[13]$.

The mechanism of resistance to quinolones proposed were due to point mutations in DNA gyrase (gyrA and gyrB) and topoisomerase IV(parC and pare genes), presence of transferable plasmid mediated quinolone resistance and mutations in genes regulating efflux pumps [16]. Another study by Lee YJ et al also reported increased resistance to parenteral use of quinolones for the treatment of Pseudomonas infections [17].

The resistance mechanism exhibited by Pseudomonas is through decreased permeability, expression of efflux systems, increased production of antibiotic inactivating enzymes and target modifications. Multidrug resistance is said to occur when a combination of more than one such mechanisms happen [18]. Although our study demonstrated resistance to fluoroquinolones only, MDR resistance is significant when there is resistance to more than one antimicrobial agent in more than three categories out of eight categories including aminoglycosides, carbapenems, cephalosporins, penicillin with beta lactamase inhibitors, monobactams, fluoroquinolones, fosfomycins and polymyxins [19].

The results of our study were further substantiated by molecular studies done by Lila $G$ et al [20]. Although their study demonstrated further resistance to more than one category of antibiotics, genotypic analysis identified significant clusters of Pulse Field Gel Electrophoresis (PFGE) patterns, which were identifiable with cross contamination of specimens isolated from Intensive Care Units (ICU), post-ICU units, neurosurgery and plastic surgery units. Certain other PFGE strains were also isolated from pulmonology, abdomen surgery and orthopedics unit.

This significantly correlates with the impact of hospital acquired infections on Pseudomonas resistance. Further analysis at the molecular level showed the presence of point mutations in the MDR phenotype resulting in multidrug resistance in Pseudomonas infections [21].
Considering the fact that hospital acquired infections contribute majorly to the antibiotic resistance in Pseudomonas infections, it is highly pertinent that stringent infection control measures are in place in order to combat these infections. There is an imminent need to develop standard protocols for case management, especially in ICU settings, so as to minimize the vulnerability towards infections. In addition, the judicious and optimized use of antibiotics is crucial for preventing antibiotic resistance. Periodic audit of prescriptions and capacity strengthening for primary care physicians should be in place to combat the menace of antibiotic resistance, so as to minimize morbidity and mortality of $P$. aeruginosa infections in the future.

\section{Conclusion}

The present study emphasized on the increased prevalence of urinary tract infections, predominantly nosocomial, resulting in Pseudomonas infections. Though our study reported a high sensitivity to Amikacin, we observed a high level of resistance to standard antipseudomonal agents like quinolones and carbepenems. There is a need for further research in exploring the mechanism of antibiotic resistance at the genetic and molecular level to give a better understanding on the prudency of antibiotic use. From the present findings, we understand that Amikacin and Gentamycin can be the proposed drug of choice for severe infections with Pseudomonas aeruginosa.

Limitation: Our study has highlighted the profile of presentation of bacteria with respect to specimen isolates and antibiotic resistance. An in-depth analysis of the clinicopathological conditions and the infection profile of the study participants could have helped substantiate the magnitude of the infectious nature of the bacteria.

\section{What the study adds to the existing knowledge?}

Although it is an established fact that urinary tract infections are predominantly caused by gram negative organisms, the present study has elicited the veracity of the infection spread through hospital sources. Further, this study has provided the basis for evaluating the antibiotic resistance to standard antipseudomonal agents, thereby focusing the need for molecular studies to evaluate antibiotic susceptibility for Pseudomonas.

\section{Declaration}

Findings: Nil; Conflict of Interest: None initiated Permission from IRB: Yes

Ethical approval - Obtained

\section{References}




\section{Original Research Article}

1. Pathogen Profile Dictionary. Available from https:// www.ppdictionary.com/bacteria/gnbac/aeruginosa.htm

2. Pollack M. Pseudomonas aeruginosa. Priciples and preactioce of infectious disease. 1995:1980-2003.

3. Pragasam AK, Raghanivedha M, Anandan S, Veeraraghavan B. Characterization of Pseudomonas aeruginosa with discrepant carbapenem susceptibility profile. Ann Clin Microbiol Antimicrob. 2016;15:12. doi:10.1186/s12941-016-0127-3.

4. Queenan AM, Shang W, Flamm R, Bush K. Hydrolysis and inhibition profiles of beta-lactamases from molecular classes $\mathrm{A}$ to $\mathrm{D}$ with doripenem, imipenem, and meropenem. Antimicrob Agents Chemother. 2010; 54(1):565-569. doi: 10.1128/AAC. 01004-09. Epub 2009 Nov 2.

5. Livermore DM. Of Pseudomonas, porins, pumps and carbapenems. J Antimicrob Chemother. 2001;47(3): 247-250. doi:10.1093/jac/47.3.247

6. Almaghrabi R, Clancy CJ, Doi Y, Hao B, Chen L, Shields RK, et al. Carbapenem-resistant Klebsiella pneumoniae strains exhibit diversity in aminoglycosidemodifying enzymes, which exert differing effects on plazomicin and other agents. Antimicrob Agents Chemother. 2014;58(8):4443-4451. doi: 10.1128/AAC. 00099-14. Epub 2014 May 27.

7. Ku K, Pogue JM, Moshos J, Bheemreddy S, Wang $\mathrm{Y}$, Bhargava A, et al. Retrospective evaluation of colistin versus tigecycline for the treatment of Acinetobacter baumannii and/or carbapenem-resistant Enterobacteriaceae infections. Am J Infect Control. 2012;40(10):983-987. doi: 10.1016/j.ajic.2011.12.014. Epub 2012 Mar 21.

8. Chander A, Raza MS. Antimicrobial susceptibility patterns of pseudomonas aeruginosa clinical isolates at a tertiary care hospital in Kathmandu, Nepal. Asian J. Pharm. Clin. Res 2013;6(Supp13):235-238.

9. Djordjevic Z, Folic MM, Zivic Z, Markovic V, Jankovic SM. Nosocomial urinary tract infections caused by Pseudomonas aeruginosa and Acinetobacter species: sensitivity to antibiotics and risk factors. Am J Infect Control.2013;41(12):1182-1187.doi:10.1016/j. ajic. 2013.02.018. Epub 2013 May 29.

10. Bassetti M, Vena A, Croxatto A, Righi E, Guery B. How to manage Pseudomonas aeruginosa infections. Drugs Context. 2018; 7: 212527. doi: 10.7573/dic. 212527. eCollection 2018.
11. Mittal R, Aggarwal S, Sharma S, Chhibber S, Harjai K. Urinary tract infections caused by Pseudomonas aeruginosa: a minireview. J Infect Public Health. 2009; 2 (3):101-111. doi: 10.1016/j.jiph.2009.08.003. Epub 2009 Sep 19.

12. Lamas Ferreiro JL, Álvarez Otero J1, González González L, Novoa Lamazares L, Arca Blanco A, Bermúdez Sanjurjo JR, et al. Pseudomonas aeruginosa urinary tract infections in hospitalized patients: Mortality and prognostic factors. PLoS One. 2017;12 (5): e0178178. doi: 10.1371/journal.pone.0178178. e Collection 2017.

13. Golia S, Suhani, Manasa S, Jyoti. Isolation of Pseudomonas aeruginosa from various clinical isolates and it antibiotic resistance pattern in a tertiary care hospital. Int J Curr Microbiol App Sci 2016;5(3):247253.doi:http://dx.doi.org/10.20546/ijcmas.2016.503.030

14.Javiya VA, Ghatak SB, Patel KR, Patel JA. Antibiotic susceptibility patterns of Pseudomonas aeruginosa at a tertiary care hospital in Gujarat, India. Indian J Pharmacol. 2008;40(5):230-234. doi: 10.4103/ 0253- 7613.44156 .

15. Livermore DM. Of Pseudomonas, porins, pumps and carbapenems. J Antimicrob Chemother. 2001;47(3): 247-250. doi:10.1093/jac/47.3.247

16. Hall RM. Mobile gene cassettes and integrons: moving antibiotic resistance genes in gram-negative bacteria. Ciba Found Symp. 1997; 207:192-202; discussion 202-205. doi:10.1002/9780470515358.ch12

17. Lee YJ, Liu HY, Lin YC, Sun KL, Chun CL, Hsueh PR. Fluoroquinolone resistance of Pseudomonas aeruginosa isolates causing nosocomial infection is correlated with levofloxacin but not ciprofloxacin use. Int J Antimicrob Agents. 2010;35(3):261-264. doi: 10.1016/j.jjantimicag.2009.11.007. Epub 2009 Dec 31.

18.Fujii A, Seki M, Higashiguchi M, Tachibana I, Kumanogoh A, Tomono K. Community-acquired, hospital-acquired, and healthcare-associated pneumonia caused by Pseudomonas aeruginosa. Respir Med Case Rep. 2014;12:30-33. doi: 10.1016/j.rmcr.2014.03.002. e Collection 2014.

19.El ZowalatyME, AlThaniAA, WebsterTJ, ElZowalat y AE, Schweizer HP, Nasrallah GK, et al. Pseudomonas aeruginosa: arsenal of resistance mechanisms, decades of changing resistance profiles, and future antimicrobial therapies. Future Microbiol. 2015; 10(10):1683-1706. doi: 10.2217/fmb.15.48. Epub 2015 Oct 6. 


\section{Original Research Article}

20. Lila G, Mulliqi G, Raka L, Kurti A, Bajrami R, Azizi E. Molecular epidemiology of Pseudomonas aeruginosa in University Clinical Center of Kosovo. Infect Drug Resist. 2018;11:2039-2046. doi: 10.2147/ IDR.S174940. eCollection 2018.
21. Domitrovic TN, Hujer AM, Perez F, Marshall SH, Hujer KM, Woc-Colburn LE, et al. Multidrug resistant Pseudomonas aeruginosa causing prosthetic valve endocarditis: a genetic-based chronicle of evolving antibiotic resistance. Open Forum Infect Dis. 2016;3(4): ofw188.doi:10.1093/ofid/ofw188. eCollection 2016 Oct

\section{How to cite this article?}

Karthick M. Pseudomonas aeruginosa: distribution and antibiotic profile of one of the ESKAPE pathogen. Trop J Path Micro 2019;5(9):678-683.doi:10.17511/jopm.2019.i09.10. 